\title{
The dielectrophoretic and travelling wave forces generated by interdigitated electrode arrays: analytical solution using Fourier series
}

\author{
Hywel Morgan $^{1}$, Alberto García Izquierdo ${ }^{1}$, David Bakewell ${ }^{1}$, \\ Nicolas G Green ${ }^{2}$ and Antonio Ramos ${ }^{2}$ \\ ${ }^{1}$ Bioelectronics Research Centre, Department Electronics and Electrical Engineering, \\ University of Glasgow, G12 8LT, UK \\ ${ }^{2}$ Departamento de Electrónica y Electromagnetismo, Facultad de Física, \\ Universidad de Sevilla, Avenida de Reina Mercedes s/n, Sevilla 41012, Spain \\ E-mail: h.morgan@elec.gla.ac.uk
}

Received 16 August 2000

\begin{abstract}
In alternating current electrokinetics, electric fields are used to generate forces on particles. Techniques have been applied for the manipulation of particles and the measurement of their dielectric properties. The fields are typically generated by microelectrode structures fabricated on planar surfaces. One particular design, using interdigitated bar electrodes, is used both in dielectrophoretic field flow fractionation and travelling wave dielectrophoresis. This paper presents a Fourier series analysis of the dielectrophoretic force on a particle generated by this type of electrode array, for both dielectrophoresis and travelling wave dielectrophoresis. Simple expressions are derived for the force at a distance of the order of the electrode spacing from the electrodes. A full analytical expression is given for the dielectrophoretic force in two dimensions. Comparisons are made with previously published experimental observations.
\end{abstract}

\section{Introduction}

Alternating current (ac) electrokinetics is the study of particle movement arising from the interaction of a non-uniform ac electric field with polarizable particles $[1,2]$. The technique is now being used for the analysis and separation of biological particles, such as cells, bacteria and viruses [3-6]. One ac electrokinetic technique is dielectrophoresis (DEP), where polarizable particles move towards or away from regions of strong electric field. DEP forces can be used in conjunction with hydrodynamic forces and gravity to separate particles using field flow fractionation (FFF) methods [7-9]. In this case, the DEP force is generated using an array of interdigitated planar electrodes that forms the bottom of a flow-through chamber. The force levitates different particles to different heights producing a vertical separation. The particles experience a drag force from a parabolic flow profile, resulting in horizontal separation along the electrode array.

Another widely used technique for particle manipulation and separation is travelling wave dielectrophoresis (twDEP). In this case particles move in a travelling electric field generated by an array of interdigitated parallel electrodes [10-13] and there is no need to pump liquid along the device in order to produce horizontal motion. The horizontal velocity of the particles (i.e. the twDEP force) depends on the effective polarizability and size of the particles. Therefore a mixture of different particles can be fractionated into 'bands' along the device, and if long arrays are used then a high degree of separation is possible [13].

For both of these techniques, the electrode arrays are similar, consisting of a large number of thin parallel bar microelectrodes (typically 10-40 $\mu \mathrm{m}$ wide) fabricated on a flat substrate (such as a glass microscope slide). A sample consisting of a suspension of particles in an electrolyte is placed on the array and a voltage applied to the microelectrodes to produce the required electrokinetic forces. The force for both DEP and twDEP depends on a number of factors, including the effective polarizability of the particle (which in turn depends on the frequency) and also the electric field. Thus knowledge 
of the electric field generated in these systems is essential for an accurate analysis and modelling of particle behaviour.

The electric field (and as a result the DEP force) has been solved analytically and numerically for a number of electrode geometries using various techniques such as point charge, charge density, finite difference, integral equation methods or Fourier series [14-18]. The forces in both DEP and twDEP cases for a parallel interdigitated array have been solved using similar numerical methods [19], as well as using analytical approximations based on Green's theorem [20] and circuit simulation methods for electrorotation electrodes [21].

In this paper we use Fourier series analysis to model the electric field from a parallel bar electrode array for two cases: first for a two-phase electrode array where only the DEP force is present and second for the case of twDEP with a four-phase electrode array. The forces in these two cases are calculated and compared with experimental results and previous solutions presented in the literature. One aim of this paper is to provide analytical expressions for the force on a particle, which can readily be compared with experimental results and used to develop models for particle movement.

\section{Basic equations and assumptions}

\subsection{Dielectrophoretic forces}

The dielectrophoretic force and the travelling wave dielectrophoretic force arise from the interaction of a non-uniform electric field with the dipole moment induced in a polarizable particle. Generally single frequency signals are used to generate the electric field, which can then be written in phasor notation as $\boldsymbol{E}=\operatorname{Re}\left[\tilde{\boldsymbol{E}} \mathrm{e}^{\mathrm{i} \omega t}\right]$, where $\mathrm{i}=\sqrt{-1}, \omega$ is the frequency of the electric field, $t$ is the time, $\operatorname{Re}[\ldots]$ indicates the real part of [...] and $\tilde{\boldsymbol{E}}$ is a general complex amplitude of the electric field.

In a uniform field, the complex amplitude of the induced dipole moment of a spherical particle is proportional to that of the electric field so that

$$
\tilde{\boldsymbol{p}}=v \alpha \tilde{\boldsymbol{E}}
$$

where $v$ is the volume of the particle and $\alpha$ is the effective polarizability. The polarizability is a complex factor that depends on the permittivity and conductivity of both the particle and the fluid, and the frequency of the applied field. For a non-uniform field, assuming that the particle diameter is much smaller than a typical distance associated with the nonuniformity, the higher order moments can be neglected and the particle can be considered to have the induced dipole moment as given by equation (1).

The time-averaged force on the dipole of the particle is [2]

$$
\langle\boldsymbol{F}\rangle=\frac{1}{2} \operatorname{Re}\left[(\tilde{\boldsymbol{p}} \cdot \nabla) \tilde{\boldsymbol{E}}^{*}\right]=\frac{1}{2} v \operatorname{Re}\left[\alpha(\tilde{\boldsymbol{E}} \cdot \nabla) \tilde{\boldsymbol{E}}^{*}\right]
$$

where $*$ indicates a complex conjugate. If the electric field has a non-uniform magnitude but no variation in phase, the force can be written as

$$
\langle\boldsymbol{F}\rangle=\frac{1}{4} v \operatorname{Re}[\alpha] \nabla|\tilde{\boldsymbol{E}}|^{2} .
$$

Wang et al [22] showed that if the electric field also has a spatially dependent phase (as in the case of twDEP), then the force expression includes an additional component due to the gradient of the phase. If the components of the electric field are written as

$$
\begin{aligned}
& E_{x}(t)=E_{x} \cos \left(\omega t+\varphi_{x}\right) \\
& E_{y}(t)=E_{y} \cos \left(\omega t+\varphi_{y}\right) \\
& E_{z}(t)=E_{z} \cos \left(\omega t+\varphi_{z}\right)
\end{aligned}
$$

the total dielectrophoretic force is

$$
\begin{aligned}
& \langle\boldsymbol{F}(t)\rangle=\frac{1}{4} v\left(\operatorname{Re}[\alpha] \nabla|\boldsymbol{E}|^{2}\right. \\
& \left.\quad+2 \operatorname{Im}[\alpha]\left(E_{x}^{2} \nabla \varphi_{x}+E_{y}^{2} \nabla \varphi_{y}+E_{z}^{2} \nabla \varphi_{z}\right)\right)
\end{aligned}
$$

where $\operatorname{Im}[. .$.$] indicates the imaginary part of [...].$

\subsection{General statements and assumptions}

For currents and frequencies typically used in ac electrokinetic systems, Maxwell's equations can be reduced to the quasielectrostatic form [23]:

$$
\begin{array}{ll}
\boldsymbol{E}=-\nabla \phi & \text { the electric field is irrotational } \\
\nabla \cdot \boldsymbol{J}+\frac{\partial \rho}{\partial t}=0 & \text { the charge conservation equation } \\
\nabla \cdot \boldsymbol{D}=\rho & \text { Gauss's law }
\end{array}
$$

where $\boldsymbol{J}$ is the current density, $\rho$ is the free charge density and $D$ is the electric flux density or the displacement vector. For a homogeneous linear dielectric with permittivity $\varepsilon$ and conductivity $\sigma, \boldsymbol{J}=\sigma \boldsymbol{E}$ and $\boldsymbol{D}=\varepsilon \boldsymbol{E}$, and the equation for the potential is

$$
\sigma \nabla^{2} \phi+\varepsilon \frac{\partial}{\partial t} \nabla^{2} \phi=0
$$

For a harmonic ac signal of frequency $\omega$, the general stationary solution is a time-dependent function given by

$$
\phi=\phi_{1} \cos \omega t+\phi_{2} \sin \omega t
$$

where both $\phi_{1}$ and $\phi_{2}$ satisfy Laplace's equation. Equation (6) is valid if the power dissipation is not high enough to create inhomogeneities in the dielectrics. In addition, the frequency must be high enough so that any double-layer effects at the electrode-electrolyte interface can be neglected [24,25]. These effects are not just those related to electrode polarization but also the generation of ac electro-osmotic fluid flow in the electrolytic medium [25, 26].

Figure 1 shows a diagram of the electrode geometry for a typical DEP or twDEP separation system. Thin electrodes are fabricated on a planar insulating substrate with width $d_{1}$ and spacing $d_{2}$ as shown. Given this particular geometry, further simplifications can be made. The electrodes are assumed to be much longer than $d_{1}$ so that the problem can be reduced to two dimensions. The electrode array is assumed to be of infinite length so that symmetry arguments can be used to reduce the problem to a unit cell, which is repeated periodically.

\subsection{Boundary conditions}

The boundary condition for the electrodes is that of a fixed potential. In this paper, two cases will be considered: a dielectrophoretic array and a travelling wave dielectrophoretic array. The two cases have different unit cells and different boundary conditions for the potential at the electrodes. However, certain boundary conditions are common to the two configurations. 


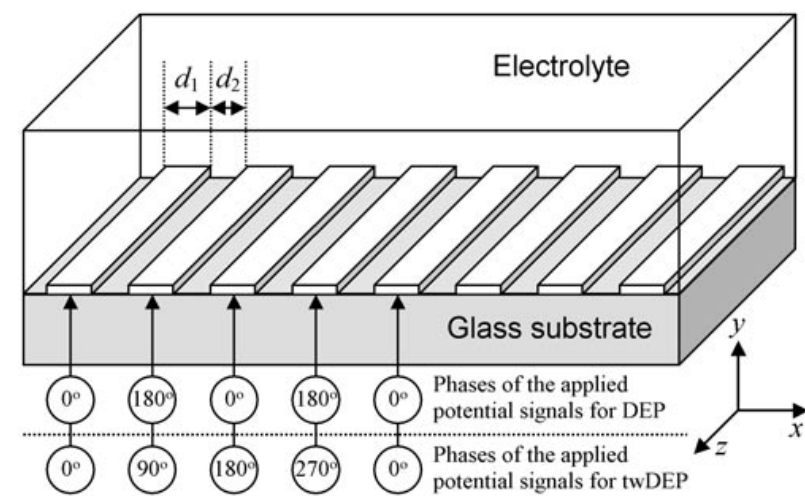

Figure 1. Diagram showing the experimental arrangement of an interdigitated electrode array used for dielectrophoretic separation devices and travelling wave dielectrophoresis. The electrodes are connected in phase sequence as shown in the diagram.

(a) The potential goes to zero as $y$ goes to infinity.

(b) Since the electrodes are much thinner than their width, we ignore the thickness so that the potential on the electrodes is specified at $y=0$.

(c) At the interface between the liquid and the glass, in the gap between the electrodes, the potential at $y=0$ satisfies

$$
\Delta\left[\sigma \frac{\partial \phi}{\partial n}+\varepsilon \frac{\partial}{\partial t} \frac{\partial \phi}{\partial n}\right]=0
$$

where $\Delta[\ldots]$ signifies the jump in [...] between the liquid and the glass of the substrate and $n$ is the normal to the surface. This equality arises from the requirement that the total current (displacement and free) across the interface is continuous. In order to obtain an analytical expression for the force, it is assumed that the potential between the electrodes is a linear function.

\section{The dielectrophoretic array}

For DEP, the phases are connected as depicted in figure 1, with electrodes connected alternately to two signals of frequency $\omega$ with phases $0^{\circ}$ and $180^{\circ}$. In this case, $\phi_{2}$ in equation (7) is zero, the phases $\varphi$ in equation (4) are independent of position and the phase related term (twDEP) in equation (5) is zero. The boundary condition at $y=0$ for the analytical solution of the electrical potential is shown in figure 2 .

\subsection{The electrical potential}

The function $\phi_{1}(x, y)$ is a solution of Laplace's equation and can be determined uniquely for $y>0$ if the boundary condition at $y=0$ is known. Since the boundary condition is periodic in $x$ with period $\lambda=2\left(d_{1}+d_{2}\right), \phi_{1}(x, 0)$ can be written as a Fourier series

$$
\phi_{1}(x, 0)=\sum_{n=1}^{\infty} A_{n} \cos \left(k_{n} x\right)
$$

where $k_{n}=2 \pi n / \lambda, n$ is an integer and $A_{n}$ are the Fourier coefficients. For convenience we have chosen $x=0$ to be at

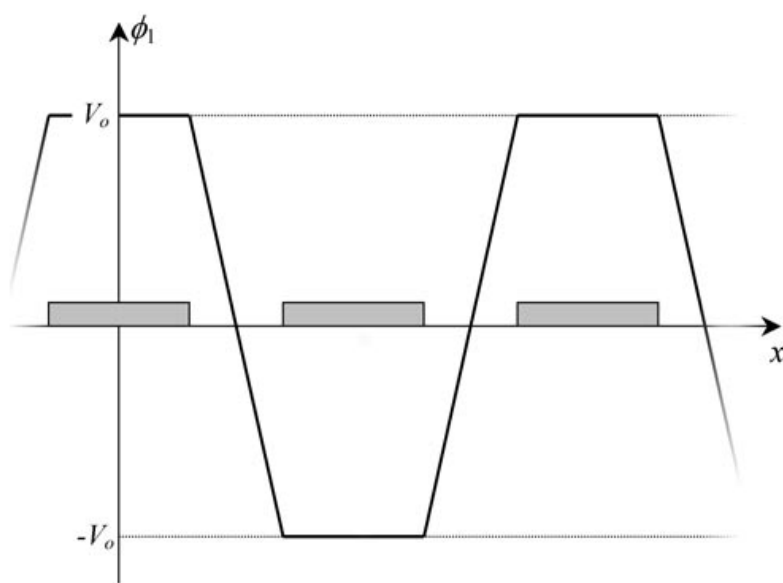

Figure 2. The boundary condition for the potential at $y=0$ used to solve the electrical potential in the system for dielectrophoresis.

the mid-point of an electrode, so that $\phi_{1}$ is an even function of $x$. The function $\phi_{1}(x, y)$ is then

$$
\phi_{1}(x, y)=\sum_{n=1}^{\infty} A_{n} \cos \left(k_{n} x\right) \mathrm{e}^{-k_{n} y} \quad y>0 .
$$

The function has odd symmetry at $x=\lambda / 4$ requiring that $\phi_{1}(\lambda / 4, y)=0$ and therefore that $\cos \left(k_{n} \lambda / 4\right)=0$. This implies that $n$ is an odd number such that $n=2 m+1$ where $m=0,1,2,3 \ldots$ and $k_{n}=[(2 m+1) \pi] / 2 d$. The coefficients $A_{n}$ are found from:

$$
\begin{aligned}
A_{n}= & \frac{2}{\lambda} \int_{0}^{\lambda} \phi_{1}(x, 0) \cos \left(k_{n} x\right) \mathrm{d} x \\
= & \frac{2 V_{o}}{d}\left[\int_{0}^{d_{1} / 2} \cos \left(k_{n} x\right) \mathrm{d} x\right. \\
& \left.+\int_{d_{1} / 2}^{d}\left(\frac{2 d-2 x}{d_{2}}\right) \cos \left(k_{n} x\right) \mathrm{d} x\right]
\end{aligned}
$$

where $d=\left(d_{1}+d_{2}\right) / 2$. Evaluating the integral

$$
A_{n}=\frac{16 V_{o} d}{\pi^{2} d_{2}(2 m+1)^{2}} \cos \left(\frac{(2 m+1) \pi d_{1}}{4 d}\right)
$$

and for the particular case $d_{1}=d_{2}=d$,

$$
A_{n}=\frac{16 V_{o}}{\pi^{2}(2 m+1)^{2}} \cos \left((2 m+1) \frac{\pi}{4}\right) .
$$

The electric potential $\phi_{1}$ is plotted in figure 3 for this case. This figure was produced using Mathematica ${ }^{\circledR}$ and shows the potential calculated from the sum of the infinite Fourier series.

\subsection{The electric field and the dielectrophoretic force}

The electric field is $\boldsymbol{E}=-\nabla \phi_{1}(x, y) \cos (\omega t)=\sum_{m=0}^{\infty}$ $\boldsymbol{E}_{m} \cos (\omega t)$ where the components of the vector $\boldsymbol{E}_{m}$ are the $m$ th terms of Fourier series:

$$
\begin{aligned}
& E_{m x}(x, y)=k_{2 m+1} A_{2 m+1} \sin \left(k_{2 m+1} x\right) \mathrm{e}^{-k_{2 m+1} y} \\
& E_{m y}(x, y)=k_{2 m+1} A_{2 m+1} \cos \left(k_{2 m+1} x\right) \mathrm{e}^{-k_{2 m+1} y}
\end{aligned}
$$

where the coefficients $A$ and $k$ are defined as above (equation (11)). Since there is no phase variation, the timeaveraged DEP force is given by equation (3)

$$
\left\langle\boldsymbol{F}_{D E P}\right\rangle=\frac{1}{4} v \operatorname{Re}[\alpha] \nabla\left(E_{x}^{2}+E_{y}^{2}\right)
$$




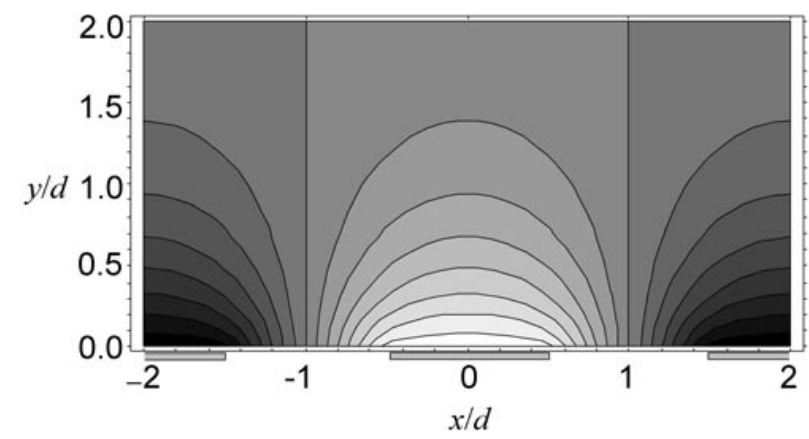

Figure 3. Contour plot of the electric potential, $\phi_{1}$, calculated from the sum of the infinite Fourier series. The grey scale extends from $-V_{o}$ (black) to $+V_{o}$ (white).

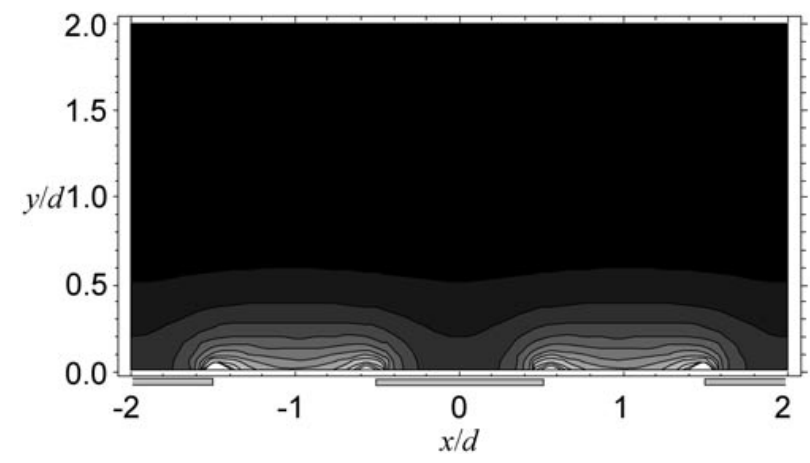

Figure 4. Contour plot of the DEP force potential, $\left(E_{x}^{2}+E_{y}^{2}\right)$. The gradient is proportional to the DEP force. The grey scale is from maximum force potential (white) to minimum (black).

where $E_{x}^{2}=\left(\sum_{m=0}^{\infty} E_{m x}\right)^{2}$ and equivalently for $E_{y}^{2}$. Figure 4 is a contour plot of the quantity $E_{x}^{2}+E_{y}^{2}$ for the case $d_{1}=d_{2}=d$. This is the DEP force potential whose gradient gives the force in equation (14) and the figure indicates how it varies in space above the electrodes. Figures 5(a) and (b) show how the positive DEP force varies above the electrodes. Figure 5(a) is a vector plot showing the direction of the force. Figure 5(b) is a contour plot of the magnitude of the force calculated on a log scale for the complete infinite series. The figure shows that for large distance from the electrode surface, the force direction is straight downwards with a constant magnitude for any given height. When particles reach a height around $d$, the direction of the force is no longer vertical, vectors point towards the electrode edges and the magnitude of the force increases rapidly.

3.2.1. The force at heights greater thand. The full expression for the force is an infinite series but this can be simplified at sufficient height above the electrodes since the higher order terms become negligible. The first term in the force expression comes from the square of $\boldsymbol{E}_{0}$ and the second comes from the inner product $\boldsymbol{E}_{1} \cdot \boldsymbol{E}_{0}$. The exponential factor in $\left|\boldsymbol{E}_{0}\right|^{2}$ is $\mathrm{e}^{-4 \pi y / \lambda}=\mathrm{e}^{-\pi y / d}$ and that in $\boldsymbol{E}_{1} \cdot \boldsymbol{E}_{0}$ is $\mathrm{e}^{-8 \pi y / \lambda}=\mathrm{e}^{-2 \pi y / d}$. For a height $y$ greater than $(\lambda / 4 \pi) \ln (10)=0.733 d$, the factor in the second term is approximately 10 times smaller than in the first. In addition, the coefficients in front of the exponential also decrease with $m$. Therefore, above a height of order $d$, all but the first term can be neglected.

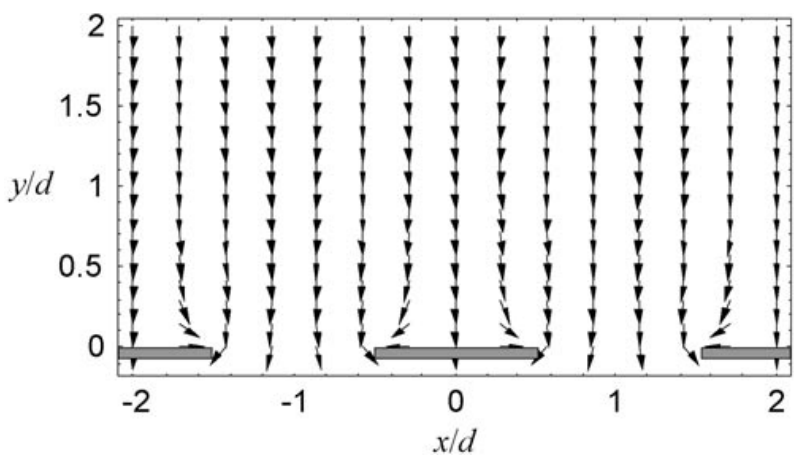

(a)

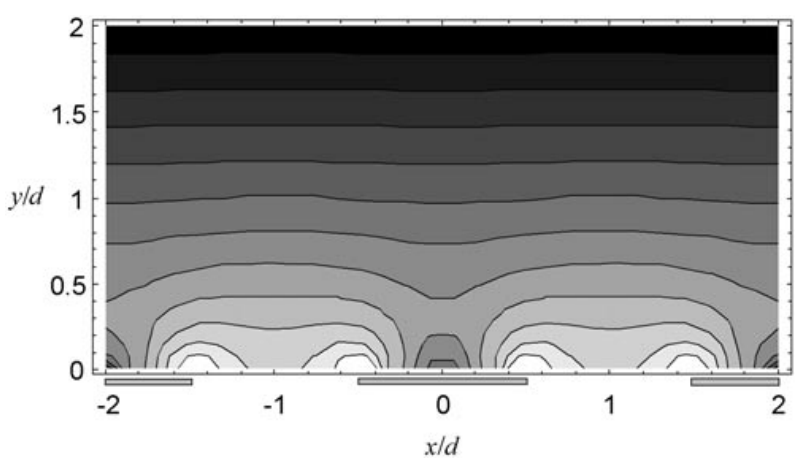

(b)

Figure 5. Plots showing the spatial variation in DEP force above the electrodes. (a) Vector plot showing the direction of the DEP force. (b) Contour plot of the magnitude of the DEP force plotted on a logarithmic, grey scale.

Considering only the first term of the series given by equation (14), the DEP force in the general case, $d_{1} \neq d_{2}$, is

$$
\left\langle\boldsymbol{F}_{D E P}\right\rangle=-16 \frac{V_{0}^{2} v}{\pi d_{2}^{2} d} \operatorname{Re}[\alpha] \cos ^{2}\left(\frac{\pi d_{1}}{4 d}\right) \mathrm{e}^{-\pi y / d} \hat{\boldsymbol{j}}
$$

with $d=\left(d_{1}+d_{2}\right) / 2$ and where $\hat{\boldsymbol{i}}, \hat{\boldsymbol{j}}$ and $\hat{\boldsymbol{k}}$ are the unit vectors.

For the particular case $d_{1}=d_{2}=d$

$$
\left\langle\boldsymbol{F}_{D E P}\right\rangle=-8 \frac{V_{0}^{2} v}{\pi d^{3}} \operatorname{Re}[\alpha] \mathrm{e}^{-\pi y / d} \hat{j} .
$$

Equations (15) and (16) are valid above $y \sim d$ and do not accurately describe the force for distances $y<d$. However, these expressions are certainly applicable for the case of negative DEP, such as in DEP-FFF systems where particles are repelled from the electrodes [7-9].

3.2.2. Full solution of the force: infinite series sum. In the particular case of $d_{1}=d_{2}=d$, a simple closed form expression can be found for the infinite series. The explicit expressions for $E_{x}$ and $E_{y}$ from equation (13) are:

$$
\begin{gathered}
E_{x}(x, y)=\sum_{n=1,3 \ldots}^{\infty} \frac{8 V_{0}}{n \pi d} \cos \left(\frac{\pi n}{4}\right) \sin \left(\frac{\pi n x}{2 d}\right) \mathrm{e}^{-k_{n} y} \\
E_{y}(x, y)=\sum_{n=1,3 \ldots}^{\infty} \frac{8 V_{0}}{n \pi d} \cos \left(\frac{\pi n}{4}\right) \cos \left(\frac{\pi n x}{2 d}\right) \mathrm{e}^{-k_{n} y}
\end{gathered}
$$


Transforming $x$ according to $x^{\prime}=x+d / 2$ these expressions become:

$$
\begin{gathered}
E_{x}(x, y)=\sum_{n=1,3 \ldots}^{\infty} \frac{4 V_{0}}{n \pi d}\left[\cos \left(\frac{\pi n x^{\prime}}{2 d}\right)\right. \\
\left.-\sin \left(\frac{\pi n}{2 d}\right) \cos \left(\frac{\pi n x^{\prime}}{2 d}\right)\right] \mathrm{e}^{-k_{n} y} \\
E_{y}(x, y)=\sum_{n=1,3 \ldots}^{\infty} \frac{4 V_{0}}{d \pi n}\left[\cos \left(\frac{\pi n x^{\prime}}{2 d}\right)\right. \\
\left.+\sin \left(\frac{\pi n}{2 d}\right) \sin \left(\frac{\pi n x^{\prime}}{2 d}\right)\right] \mathrm{e}^{-k_{n} y}
\end{gathered}
$$

which can be written in closed form as [27]:

$$
\begin{aligned}
& E_{x}(x, y)=\frac{2 V_{0}}{\pi d}\left[\tan ^{-1}\left(\frac{\sin \hat{x}}{\sinh \hat{y}}\right)-\tan ^{-1}\left(\frac{\cos \hat{x}}{\sinh \hat{y}}\right)\right] \\
& E_{y}(x, y)=\frac{V_{0}}{d \pi}\left[\ln \left(\frac{\cosh \hat{y}+\cos \hat{x}}{\cosh \hat{y}-\cos \hat{x}} \frac{\cosh \hat{y}+\sin \hat{x}}{\cosh \hat{y}-\sin \hat{x}}\right)\right]
\end{aligned}
$$

where $\hat{y}=\pi y / 2 d$ and $\hat{x}=\pi x^{\prime} / 2 d=\pi x / 2 d+\pi / 4$. The force is obtained from the derivatives of the electric field components:

$$
\begin{aligned}
& E_{x, x}(x, y)=\frac{\partial E_{x}}{\partial x}=-\frac{\partial E_{y}}{\partial y}=-E_{y, y}(x, y) \\
& =\frac{2 V_{0} \sinh \hat{y}}{d^{2}}\left[\frac{\cos \hat{x}}{\cosh 2 \hat{y}-\cos 2 \hat{x}}+\frac{\sin \hat{x}}{\cosh 2 \hat{y}+\cos 2 \hat{x}}\right] \\
& E_{x, y}(x, y)=\frac{\partial E_{x}}{\partial y}=\frac{\partial E_{y}}{\partial x}=E_{y, x}(x, y) \\
& =\frac{2 V_{0} \cosh \hat{y}}{d^{2}}\left[\frac{\cos \hat{x}}{\cosh 2 \hat{y}+\cos 2 \hat{x}}-\frac{\sin \hat{x}}{\cosh 2 \hat{y}-\cos 2 \hat{x}}\right]
\end{aligned}
$$

Expanding the gradient in equation (14) as

$$
\begin{aligned}
& \nabla\left(E_{x}^{2}+E_{y}^{2}\right)=2 \boldsymbol{u}_{x}\left(E_{x} E_{x, x}+E_{y} E_{y, x}\right) \\
& \quad+2 \boldsymbol{u}_{y}\left(E_{x} E_{y, x}+E_{y} E_{y, y}\right)
\end{aligned}
$$

and using the expressions for the derivatives (equations (20)), the $x$ - and $y$-components of the gradient can be obtained. This enables the DEP force to be calculated for a particle at an arbitrary point above the electrodes. This analytical solution can be used to calculate for example the trajectory of a Brownian particle using the Langevin equation.

\subsection{Examples and comparison with experiments}

For the particular case of a homogeneous spherical particle suspended in a liquid, the effective polarizability $\alpha$ is equal to $3 \varepsilon_{m} f_{c m}$, where $\varepsilon_{m}$ is the permittivity of the liquid and $f_{c m}$ is the complex Clausius-Mossotti factor [1,2]. The real part of $f_{c m}$ is bounded by +1 and $-1 / 2$ and the imaginary part is bounded by $+3 / 4$ and $-3 / 4$. For the case $d_{1}=d_{2}=d$, the levitation height can be found by balancing the dielectrophoretic force with the gravitational force $\boldsymbol{F}_{G}=\Delta \rho v \boldsymbol{g}$ giving

$$
h=\frac{d}{\pi} \ln \left[-\frac{24 V_{0}^{2} \varepsilon_{m} \operatorname{Re}\left[f_{C M}\right]}{\pi d^{3} \Delta \rho g}\right] .
$$

This assumes that the particle is experiencing negative dielectrophoresis, so that $\operatorname{Re}\left[f_{c m}\right]<0$ and the particle is repelled from the electrodes. For a $1 \mu \mathrm{m}$ diameter particle suspended in water on an electrode array with $d=20 \mu \mathrm{m}$ and $V_{0}=5 \mathrm{~V}$, taking $\operatorname{Re}\left[f_{c m}\right]=-1 / 2$ with a particle density twice that of water, the levitation height is $43 \mu \mathrm{m}$. Since the force obeys an exponential law, this height does not change significantly with particle density. For example, for a particle with a density three times that of water the equilibrium height is $38.5 \mu \mathrm{m}$ and for 1.5 times the density of water the height is $47.4 \mu \mathrm{m}$.

For the same $1 \mu \mathrm{m}$ particle, the force far from the electrodes is

$$
\left\langle\boldsymbol{F}_{D E P}\right\rangle=-8.75 \times 10^{-12} \operatorname{Re}\left[f_{c m}\right] \mathrm{e}^{-\pi y / d} \hat{\boldsymbol{j}} \quad \mathrm{N} .
$$

For the case of DEP field flow fractionation, Markx et al [7] calculated the DEP force on a levitated particle from numerical simulation of the electric field using finite element methods. Huang et al [8] and Wang et al [9] deduced an analytical expression for the DEP force from a solution of the potential using Green's method [20]. Direct comparison of our expression for the DEP force, equation (16) with the data presented in [7] is summarized in table 1. The agreement is excellent, provided that the applied voltage is sufficient to levitate the particles to a height greater than $d$. Also, the analytical expression for the DEP force given in $[8,9]$ is within $7 \%$ of that given by equation (16). It should be noted that equation (16) gives the DEP force when the voltage on each electrode is defined to have an amplitude of $V_{0}$, i.e. the potential difference between two consecutive electrodes is $2 V_{0}$.

\section{The travelling wave array}

In a four-phase travelling wave electrode array, each applied signal has an amplitude of $V_{0}$, and an angular frequency of $\omega$. The voltage on consecutive electrodes is phase shifted by $90^{\circ}$ as shown in figure 1 , so that the travelling wave moves in the positive direction. The wave has a wavelength $\lambda$ equal to the distance between every fourth electrode, i.e. $\lambda=4\left(d_{1}+d_{2}\right)=8 d$.

\subsection{The electrical potential}

The electrostatic potential $\phi$ is the superposition of the two functions: $\phi=\phi_{1} \cos \omega t+\phi_{2} \sin \omega t$. The functions $\phi_{1}$ and $\phi_{2}$ have the boundary conditions specified in figure 6 . The amplitude of $\phi_{2}$ is the same as $\phi_{1}$ but shifted $\lambda / 4$ in the $x$-direction: $\phi_{2}(x, y)=\phi_{1}(x-\lambda / 4, y)$.

Again, the function $\phi_{1}$ is determined completely for $y>0$ given the boundary condition at $y=0$. Because of the periodicity of the boundary condition, $\phi_{1}(x, 0)$ is written as the Fourier series

$$
\phi_{1}(x, 0)=\sum_{n=1}^{\infty} A_{n} \cos \left(k_{n} x\right)
$$

where $k_{n}=2 \pi n / \lambda$ and $\phi_{1}(x, 0)$ is again chosen to be an even function of $x$. Solving Laplace's equation, the function $\phi_{1}(x, y)$ for $y>0$ is

$$
\phi_{1}(x, y)=\sum_{n=1}^{\infty} A_{n} \cos \left(k_{n} x\right) \mathrm{e}^{-k_{n} y} .
$$


Table 1. A comparison of the experimentally determined levitation heights for $6 \mu \mathrm{m}$ diameter latex beads reported in [7], with the levitation heights calculated from equation (22).

\begin{tabular}{|c|c|c|c|c|c|c|}
\hline \multirow{2}{*}{$\begin{array}{l}\text { Electrode } \\
\text { spacing } d(\mu \mathrm{m})\end{array}$} & \multicolumn{6}{|c|}{$V_{0}=2 \mathrm{~V}\left(4 \mathrm{~V}\right.$ pk to pk) $V_{0}=3 \mathrm{~V}(6 \mathrm{~V}$ pk to $\mathrm{pk}) V_{0}=4 \mathrm{~V}(8 \mathrm{~V}$ pk to pk $)$} \\
\hline & $h(\exp )$ & $h$ (theory) & $h(\exp )$ & $h$ (theory) & $h(\exp )$ & $h$ (theory) \\
\hline 40 & 70 & 72 & 80 & 82 & 88 & 92 \\
\hline 20 & 48 & 50 & 55 & 55 & 62 & 59 \\
\hline 10 & 35 & 32 & 40 & 34 & - & - \\
\hline
\end{tabular}

As for the dielectrophoretic array, symmetry arguments require that $\phi_{1}(\lambda / 4, y)=0$ implying that $\cos \left(k_{n} \lambda / 4\right)=0$ and $k_{n}=(2 m+1) 2 \pi / \lambda$ where $m=0,1,2,3, \ldots$. Taking into account the $\lambda / 4$ shift in $x$ with respect to $\phi_{1}(x, y)$, gives the following expression for the potential $\phi_{2}(x, y)$

$$
\begin{aligned}
& \phi_{2}(x, y)=\sum_{\substack{n=1 \\
n \text { odd }}}^{\infty} A_{n} \cos \left(k_{n}(x-\lambda / 4)\right) \mathrm{e}^{-k_{n} y} \\
& =\sum_{m=0}^{\infty}(-1)^{m} A_{2 m+1} \sin \left(\frac{(2 m+1) 2 \pi}{\lambda} x\right) \mathrm{e}^{-(2 m+1)(2 \pi / \lambda) y} .
\end{aligned}
$$

The complete potential is therefore:

$$
\begin{gathered}
\phi(x, y, t)=\sum_{m=0}^{\infty} A_{2 m+1}\left[\cos \left(\frac{(2 m+1) 2 \pi}{\lambda} x\right) \cos (\omega t)\right. \\
\left.+(-1)^{m} \sin \left(\frac{(2 m+1) 2 \pi}{\lambda} x\right) \sin (\omega t)\right] \mathrm{e}^{-[(2 m+1) 2 \pi / \lambda] y} \\
=\sum_{m=0}^{\infty} A_{2 m+1} \cos \left(\frac{(2 m+1) 2 \pi}{\lambda} x-(-1)^{m} \omega t\right) \mathrm{e}^{-[(2 m+1) 2 \pi / \lambda] y} .
\end{gathered}
$$

The potential is the superposition of waves propagating alternately in the positive or negative $x$-direction depending on whether $m$ is even or odd, respectively [18]. As for the previous case (section 3.2.1), at heights greater than $(\lambda / 4 \pi) \ln (10)=1.466 d$ there is a pure propagating wave in the $x$-direction (corresponding to $m=0$ ), since at this height higher order terms are negligible.

The Fourier coefficients are found from

$$
\begin{aligned}
& A_{2 m+1}=\frac{2}{\lambda} \int_{0}^{\lambda} \phi_{1}(x, 0) \cos \left(k_{2 m+1} x\right) \mathrm{d} x \\
& =\frac{8 V_{0}}{\lambda}\left[\int_{0}^{d_{1} / 2} \cos \left(\frac{(2 m+1) \pi}{4 d} x\right) \mathrm{d} x\right. \\
& \left.\quad+\int_{d_{1} / 2}^{d_{2}+d_{1} / 2}\left(\frac{d_{1}+2 d_{2}-2 x}{d_{2}}\right) \cos \left(\frac{(2 m+1) \pi}{4 d} x\right) \mathrm{d} x\right]
\end{aligned}
$$

which gives

$$
\begin{aligned}
A_{2 m+1} & =\frac{16 V_{0} d}{(2 m+1)^{2} \pi^{2} d_{2}}\left[\cos \left(\frac{(2 m+1) \pi d_{1}}{8 d}\right)\right. \\
- & \left.\cos \left(\frac{(2 m+1) \pi\left(d_{1}+2 d_{2}\right)}{8 d}\right)\right] .
\end{aligned}
$$

For the case $d_{1}=d_{2}=d$ this simplifies to

$$
\begin{aligned}
A_{2 m+1} & =\frac{16 V_{0}}{(2 m+1)^{2} \pi^{2}}\left[\cos \left(\frac{(2 m+1) \pi}{8}\right)\right. \\
- & \left.\cos \left(\frac{(2 m+1) 3 \pi}{8}\right)\right] .
\end{aligned}
$$

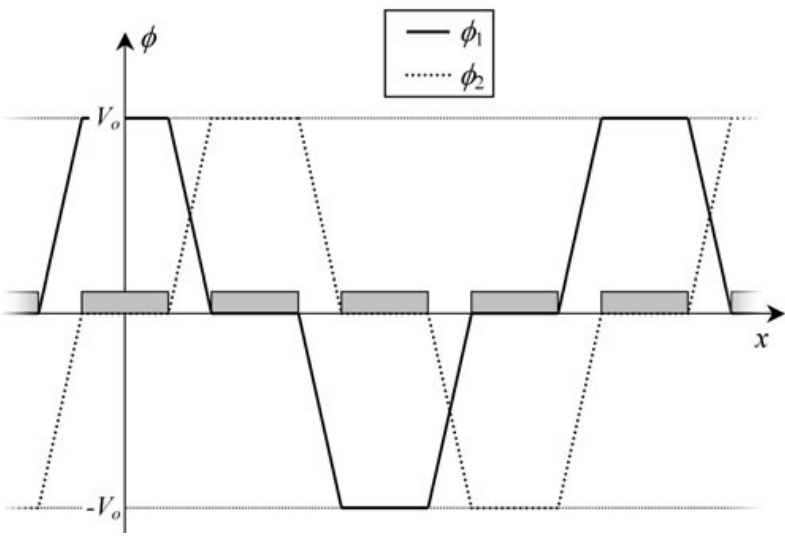

Figure 6. The boundary conditions for the potential functions $\phi_{1}$ and $\phi_{2}$ for the travelling wave dielectrophoresis array. The amplitude of $\phi_{2}$ is the same as $\phi_{1}$ but shifted $\lambda / 4$ in the $x$-direction.

\subsection{The electric field and the dielectrophoretic forces}

From $\boldsymbol{E}=-\nabla \phi_{1}(x, y) \cos (\omega t)-\nabla \phi_{2}(x, y) \sin (\omega t)$, the electric field can be written as the series $\boldsymbol{E}(x, y, t)=\sum_{m=0}^{\infty} \boldsymbol{E}_{m}$ where

$$
\begin{aligned}
& E_{m x}(x, y, t)=\frac{(2 m+1) \pi}{4 d} A_{2 m+1} \sin \left(\frac{(2 m+1) \pi}{4 d} x\right. \\
& \left.-(-1)^{m} \omega t\right) \mathrm{e}^{-[(2 m+1) \pi / 4 d] y} \\
& E_{m y}(x, y, t)=\frac{(2 m+1) \pi}{4 d} A_{2 m+1} \cos \left(\frac{(2 m+1) \pi}{4 d} x\right. \\
& \left.-(-1)^{m} \omega t\right) \mathrm{e}^{-[(2 m+1) \pi / 4 d] y} .
\end{aligned}
$$

Each term, $\boldsymbol{E}_{m}(x, y, t)$, of this series is a vector that for fixed $(x, y)$ rotates clock-wise ( $m$ odd) or anti-clockwise ( $m$ even). Alternatively, each $\boldsymbol{E}_{m}$ can be viewed as a wave that travels in the positive $x$-direction if $m$ is even and in the negative $x$-direction if $m$ is odd [18].

4.2.1. The force at heights greater than 1.5d. The full expression for the force can be simplified at sufficient height above the electrodes since higher order terms become negligible. As for the potential, at heights $y$ greater than $(\lambda / 4 \pi) \ln (10)=1.466 d$ all but the first term can be neglected. The electric field components are then:

$$
\begin{aligned}
& E_{x}(x, y, t)=\frac{\pi}{4 d} A_{1} \mathrm{e}^{-(\pi / 4 d) y} \cos \left(\omega t-\frac{\pi}{4 d} x-\frac{\pi}{2}\right) \\
& E_{y}(x, y, t)=\frac{\pi}{4 d} A_{1} \mathrm{e}^{-(\pi / 4 d) y} \cos \left(\omega t-\frac{\pi}{4 d} x\right) .
\end{aligned}
$$


The components of the DEP force are

$$
\begin{aligned}
& \left\langle F_{x}(t)\right\rangle=-v \operatorname{Im}[\alpha]\left(\frac{\pi}{4 d}\right)^{3} A_{1}^{2} \mathrm{e}^{-(\pi / 2 d) y} \\
& \left\langle F_{y}(t)\right\rangle=-v \operatorname{Re}[\alpha]\left(\frac{\pi}{4 d}\right)^{3} A_{1}^{2} \mathrm{e}^{-(\pi / 2 d) y}
\end{aligned}
$$

For the particular case of $d_{1}=d_{2}=d$

$$
A_{1}=\frac{16 V_{0}}{\pi^{2}}\left[\cos \left(\frac{\pi}{8}\right)-\cos \left(\frac{3 \pi}{8}\right)\right]=0.877354 V_{0}
$$

and

$$
\begin{aligned}
& \left\langle F_{x}(t)\right\rangle=-\frac{\pi^{3}}{64 d^{3}} v \operatorname{Im}[\alpha] A_{1}^{2} \mathrm{e}^{-(\pi / 2 d) y} \\
& =-0.372923 \frac{v V_{0}^{2}}{d^{3}} \operatorname{Im}[\alpha] \mathrm{e}^{-(\pi / 2 d) y} \\
& \left\langle F_{y}(t)\right\rangle=-0.372923 \frac{v V_{0}^{2}}{d^{3}} \operatorname{Re}[\alpha] \mathrm{e}^{-(\pi / 2 d) y} .
\end{aligned}
$$

It can be seen from these expressions that the $x$-component of the force is proportional to the imaginary part of the ClausiusMossotti factor, and gives rise to the travelling wave movement of the particle. The $y$-component is proportional to the real part of the Clausius-Mossotti factor and is responsible for levitation of the particle. It should be emphasized that these expressions are only valid from $y=1.5 d$ to infinity. Closer to the electrodes, higher order effects must be taken into account.

4.2.2. Higher order terms in the force expressions. In order to expand the problem to include higher order terms in the DEP force (equation (5)), some manipulation is required. We write the electric field components as $E_{x}(t)=C_{x} \cos (\omega t)+D_{x} \sin (\omega t)$, with a similar expression for $E_{y}$. The coefficients $C_{x}, C_{y}, D_{x}$ and $D_{y}$ are obtained from the corresponding Fourier series:

$$
\begin{aligned}
C_{x}= & \sum_{m=0}^{\infty} \frac{(2 m+1) \pi}{4 d} A_{2 m+1} \sin \left(\frac{(2 m+1) \pi}{4 d} x\right) \\
& \times \mathrm{e}^{-[(2 m+1) \pi / 4 d] y} \\
D_{x}= & -\sum_{m=0}^{\infty} \frac{(2 m+1) \pi}{4 d} A_{2 m+1}(-1)^{m} \cos \left(\frac{(2 m+1) \pi}{4 d} x\right) \\
& \times \mathrm{e}^{-[(2 m+1) \pi / 4 d] y} \\
C_{y}= & \sum_{m=0}^{\infty} \frac{(2 m+1) \pi}{4 d} A_{2 m+1} \cos \left(\frac{(2 m+1) \pi}{4 d} x\right) \\
& \times \mathrm{e}^{-[(2 m+1) \pi / 4 d] y} \\
D_{y}= & \sum_{m=0}^{\infty} \frac{(2 m+1) \pi}{4 d} A_{2 m+1}(-1)^{m} \sin \left(\frac{(2 m+1) \pi}{4 d} x\right) \\
& \times \mathrm{e}^{-[(2 m+1) \pi / 4 d] y} .
\end{aligned}
$$

The gradient terms in the force can be written as

$$
\nabla|\boldsymbol{E}|^{2}=\nabla\left(C_{x}^{2}+D_{x}^{2}+C_{y}^{2}+D_{y}^{2}\right)
$$

and

$$
\begin{aligned}
& E_{x}^{2} \nabla \varphi_{x}=D_{x} \nabla C_{x}-C_{x} \nabla D_{x} \\
& E_{y}^{2} \nabla \varphi_{y}=D_{y} \nabla C_{y}-C_{y} \nabla D_{y} .
\end{aligned}
$$

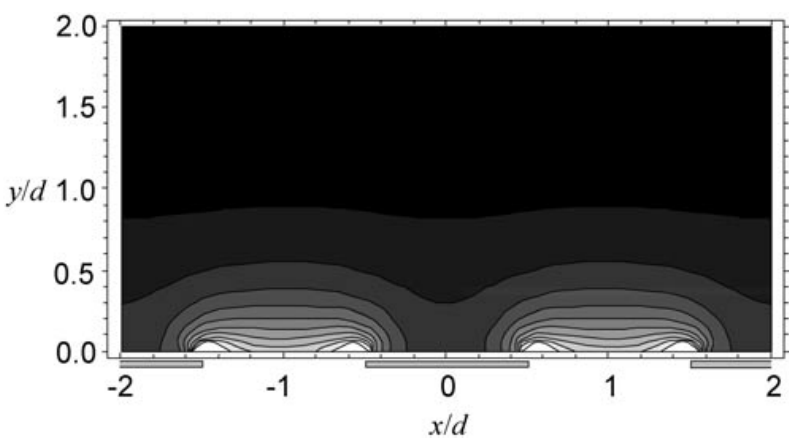

Figure 7. Contour plot of the DEP force potential in a travelling wave device, $|\boldsymbol{E}|^{2}$. The grey scale is from maximum force potential (white) to minimum (black).

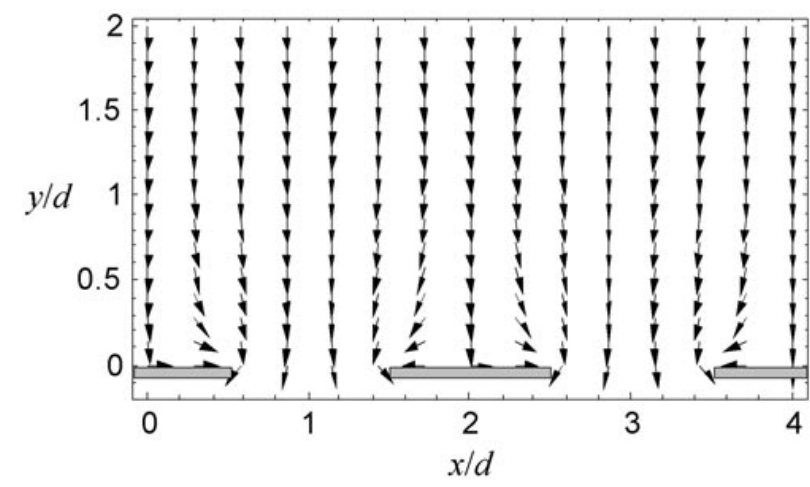

(a)

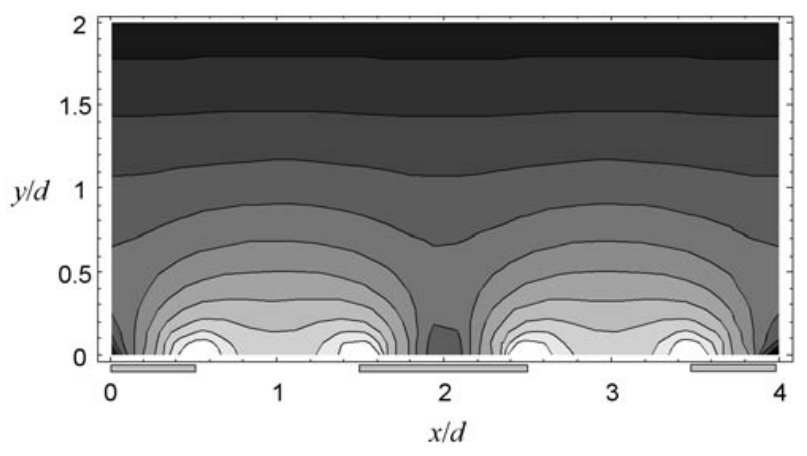

(b)

Figure 8. Plots showing the spatial variation in DEP force above the twDEP electrodes. (a) Vector plot showing the direction of the force. (b) Contour plot of the magnitude of the force plotted on a logarithmic, grey scale.

Using these equations, the expression for the complete DEP force in two dimensions is

$$
\begin{aligned}
& \langle\boldsymbol{F}\rangle=\frac{1}{4} v\left(\operatorname{Re}[\alpha] \nabla\left(C_{x}^{2}+C_{y}^{2}+D_{x}^{2}+D_{y}^{2}\right)\right. \\
& \left.\quad+2 \operatorname{Im}[\alpha]\left(D_{x} \nabla C_{x}-C_{x} \nabla D_{x}+D_{y} \nabla C_{y}-C_{y} \nabla D_{y}\right)\right) .
\end{aligned}
$$

The first term in this equation, the DEP component, is the same as for the dielectrophoretic array case, with a potential given by $|\boldsymbol{E}|^{2}=C_{x}^{2}+D_{x}^{2}+C_{y}^{2}+D_{y}^{2}$. A contour plot of this potential is shown in figure 7 for the case $d_{1}=d_{2}=d$. The DEP force is perpendicular to the iso-lines and a plot of the force is shown in figure 8 as (a) separate direction and (b) magnitude.

The twDEP component has a different behaviour that can be illustrated as follows. The functions $C_{x}, C_{y}, D_{x}$ and $D_{y}$ 


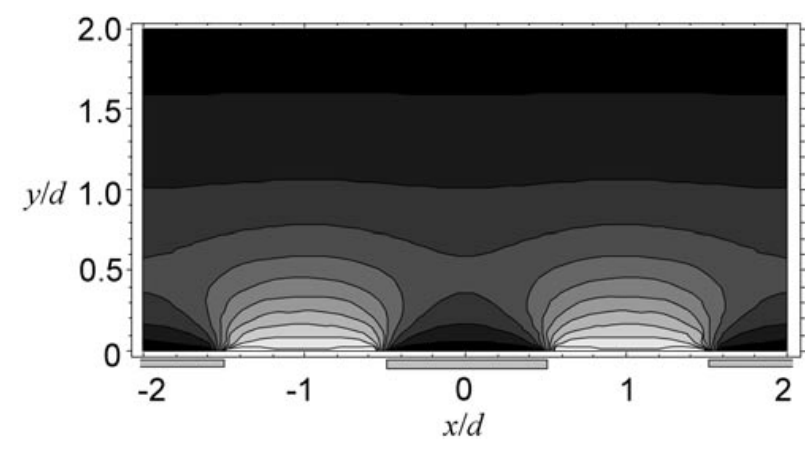

Figure 9. Contour plot of $\psi$. The lines of constant $\psi$ are parallel to the direction of the travelling wave force (as shown by the vectors of figure 10(a)).

obey the following equalities:

$$
\frac{\partial C_{x}}{\partial x}=-\frac{\partial C_{y}}{\partial y} \quad \frac{\partial C_{y}}{\partial x}=\frac{\partial C_{x}}{\partial y}
$$

since they are the partial derivatives of $\phi_{1}$ and $\phi_{2}$. Taking this into account, it can be shown that

$$
\begin{aligned}
& D_{x} \nabla C_{x}-C_{x} \nabla D_{x}+D_{y} \nabla C_{y}-C_{y} \nabla D_{y} \\
& \quad=\nabla \times\left[\left(C_{x} D_{y}-C_{y} D_{x}\right) \hat{k}\right] .
\end{aligned}
$$

In two dimensions, the function $\psi=C_{x} D_{y}-C_{y} D_{x}$ is similar to a two-dimensional stream function for the twDEP force component. The lines of constant $\psi$ are parallel to the force vectors and figure 9 shows a contour plot of $\psi$ for the case $d_{1}=d_{2}=d$, i.e. the twDEP component direction. In the absence of any other forces, including the DEP component, the contour lines in this figure indicate the path of the particle. This can be seen in figure 10, which shows the twDEP component of the force as (a) separate direction and (b) magnitude plots. Again for $y>d$ the force is uniform but for lower heights the particle trajectory is no longer a simple translational movement.

\subsection{Examples}

For the case of the homogeneous spherical particle (as in section 3.2.3), with an applied potential of $5 \mathrm{~V}$, there are certain frequencies at which the particle levitates and experiences a twDEP force since the imaginary part of $f_{c m}$ is non-zero. Taking typical values of -0.1 for the real and -0.65 for the imaginary parts of $f_{c m}$, and assuming that the particle is sufficiently high so that the far-field approximation can be used, the corresponding forces are: ]

$$
\begin{array}{ll}
\left\langle F_{x}\right\rangle=8.330 \times 10^{-13} \mathrm{e}^{-\pi y / 2 d} & \mathrm{~N} \\
\left\langle F_{y}\right\rangle=8.330 \times 10^{-13} \mathrm{e}^{-\pi y / 2 d} & \mathrm{~N} .
\end{array}
$$

For a particle with a density twice that of water, the equilibrium levitation height is $41 \mu \mathrm{m}$. At this height the velocity of the particle can be calculated (from Stoke's formula, $\left.v_{x}=F_{x} /(6 \pi \eta a)\right)$ to be $3.5 \mu \mathrm{m} \mathrm{s}^{-1}$.

The relationship between experimental parameters and the movement of the particle in the regime far from the electrodes $(y>1.5 d)$ can be examined further. As for the previous case, the levitation height of the particle can be determined

$$
h=\frac{2 d}{\pi} \ln \left[-\frac{0.3729 V_{0}^{2} 3 \varepsilon_{m} \operatorname{Re}\left[f_{C M}\right]}{d^{3} \Delta \rho g}\right] .
$$

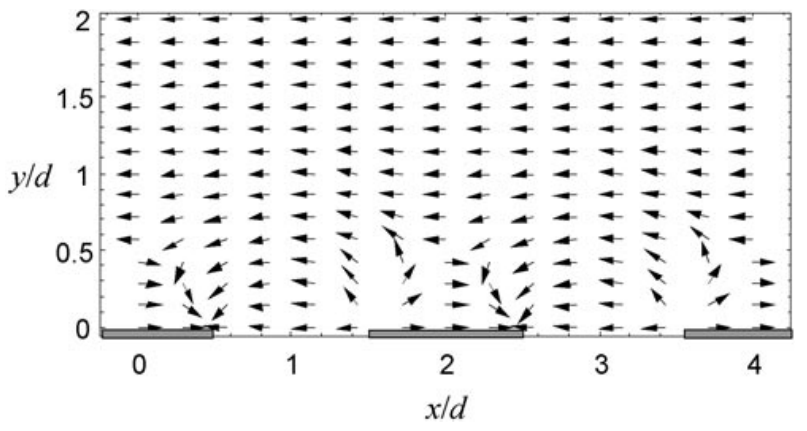

(a)

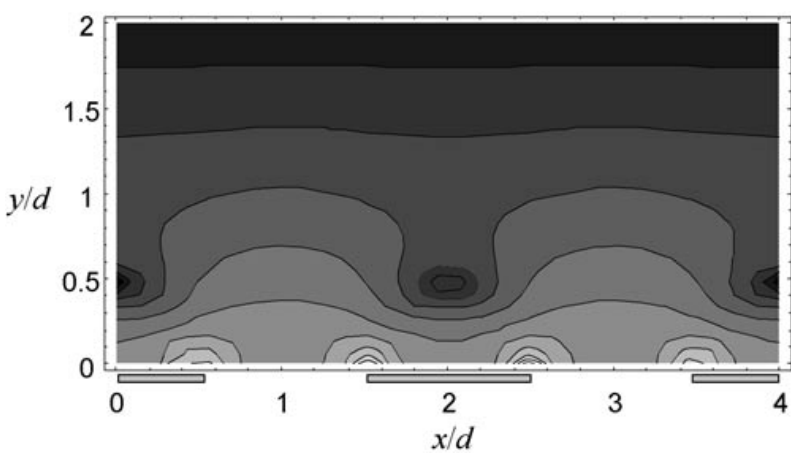

(b)

Figure 10. Plots showing the spatial variation in travelling wave force above the twDEP electrodes. (a) Vector plot showing the direction of the travelling wave force. (b) Contour plot of the magnitude of the force plotted on a logarithmic, grey scale.

Substituting this into equation (35) and equating with the Stoke's drag force gives the velocity of the particle

$$
v_{x}=\frac{2}{9} \frac{a^{2} \Delta \rho g}{\eta} \frac{\operatorname{Im}\left[f_{C M}\right]}{\operatorname{Re}\left[f_{C M}\right]} .
$$

This signifies that once the particle has reached a levitation height greater than $1.5 d$, the velocity of the particle is independent of the applied voltage. For a given particle, the velocity only depends on the frequency of the applied signal, through the ratio of the imaginary and real parts of the Clausius-Mossotti factor. To increase the velocity, the real part of the Clausius-Mossotti factor can be reduced but this would also reduce the levitation height. Experimental results [28] confirm that the twDEP velocity is independent of the voltage when the voltage is high enough to levitate the particle above $y \sim d$.

\section{Conclusion}

Fourier series analysis of the solution to Laplace equation has been used to obtain an analytical expression for the DEP and twDEP forces in an interdigitated electrode array. The solution was obtained assuming a linear potential function between neighbouring electrodes. A simple expression has been derived for the DEP and twDEP force at a distance of the order of the electrode spacing from the surface of the device. A full analytical solution for the $x$ and $y$ components of the force has been obtained for the DEP electrode array. Comparisons with previously published experimental data on DEP and twDEP levitation show good agreement with the analytical solutions. 


\section{Acknowledgments}

The authors would like to acknowledge the support of the Engineering and Physical Sciences Research Council, UK (grant no GR/M31255), the European Union for the award of a Marie Curie fellowship (contract no BIO4-CT98-5010 (DG12SSMI)) to N G Green and the Spanish Government Agency Dirección General de Ciencia y Tecnología under contract PB96-1375. They would also like to thank the University of Glasgow and the CVCP Overseas Research Students Awards Scheme for a Scholarship for D Bakewell.

\section{References}

[1] Pohl H A 1978 Dielectrophoresis (Cambridge: Cambridge University Press)

[2] Jones T B 1995 Electromechanics of Particles (Cambridge: Cambridge University Press)

[3] Becker F F, Wang X-B, Huang Y, Pethig R, Vykoukal J and Gascoyne P R C 1995 Proc. Natl Acad. Sci. USA. 92 $860-4$

[4] Markx G H, Huang Y, Zhou X F and Pethig R 1994 Microbiology-UK 140 585-91

[5] Green N G and Morgan H 1997 J. Phys. D: Appl. Phys. 30 L41-4

[6] Morgan H, Hughes M P and Green N G 1999 Biophys J. 77 $516-25$

[7] Markx G H, Pethig R and Rousselet J 1997 J. Phys. D: Appl. Phys. 30 2470-7

[8] Huang Y, Wang X-B, Becker F F and Gascoyne P R C 1997 Biophysical J. 73 1118-29

[9] Wang X-B, Vykoukal J, Becker F F and Gascoyne P R C 1998 Biophysical J. 74 2689-701
[10] Hagedorn R, Fuhr G, Muller T and Gimsa J 1992 Electrophoresis 13 49-54

[11] Huang Y, Wang X-B, Tame J and Pethig R 1993 J. Phys. D: Appl. Phys. 26 312-22

[12] Talary M S, Burt J P H, Tame J A and Pethig R 1996 J. Phys. D: Appl. Phys. 29 2198-203

[13] Morgan H, Green N G, Hughes M P, Monaghan W and Tan T C 1997 J. Micro. Microeng. 7 65-70

[14] Schnelle T, Hagedorn R, Fuhr G, Fiedler S and Müller T 1993 Biochim. Biophys. Acta. 1157 127-40

[15] Wang X-B, Huang Y, Burt J P H, Markx G H and Pethig R 1993 J. Phys. D: Appl. Phys. 26 1278-85

[16] Martinez G and Sancho M 1983 Am. J. Phys. 51 170-4

[17] Pethig R, Huang Y, Wang X-B and Burt J P H 1992 J. Phys. D: Appl. Phys. 25 881-8

[18] Masuda S, Washizu M and Iwadare M 1987 IEEE Trans. Ind. Appls. IA-23 474-80

[19] Hughes M P, Pethig R and Wang X-B 1996 J. Phys. D: Appl. Phys. 29 474-82

[20] Wang X-J, Wang X-B, Becker F F and Gascoyne P R C 1996 J. Phys. D: Appl. Phys. 29 1649-60

[21] Holzel R 1993 J. Phys. D: Appl. Phys. 26 2112-16

[22] Wang X-B, Huang Y, Becker F F and Gascoyne P R C 1994 J. Phys. D: Appl. Phys. 27 1571-4

[23] Melcher J R 1981 Continuum Electromechanics (Cambridge, MA: MIT Press)

[24] Sluyters-Rehbach M and Sluyters J H 1970 Electroanalytical Chemistry vol 4, ed A J Bard (New York: Dekker) ch 1

[25] Ramos A, Morgan H, Green N G and Castellanos A 1999 J. Colloid Interface Sci. 217 420-2

[26] Green N G, Ramos A, Gonzalez A, Morgan H and Castellanos A 2000 Phys. Rev. E 61 4011-18

[27] Gradshteyn I S and Ryzhik I M 1994 Table of Integrals, Series, and Products 5th edn, ed A Feffrey (London: Academic)

[28] Wang X-B, Huang Y, Wang X, Becker F F and Gascoyne P R C 1997 Biophys. J. 72 1887-99 